\title{
Protée
}

\section{Pour une approche sémiotique des pratiques éthiques}

\section{Maria Giulia Dondero}

Volume 36, numéro 2, automne 2008

Éthique et sémiotique du sujet

URI : https://id.erudit.org/iderudit/019015ar

DOI : https://doi.org/10.7202/019015ar

Aller au sommaire du numéro

Éditeur(s)

Département des arts et lettres - Université du Québec à Chicoutimi

ISSN

0300-3523 (imprimé)

1708-2307 (numérique)

Découvrir la revue

Citer ce document

Dondero, M. G. (2008). Pour une approche sémiotique des pratiques éthiques. Protée, 36(2), 5-10. https://doi.org/10.7202/019015ar d'utilisation que vous pouvez consulter en ligne.

https://apropos.erudit.org/fr/usagers/politique-dutilisation/ 
PRÉSEN TATIO N

\title{
PO UR UNE APPRO CHE SÉMIO TIQ UE DES PRATIQ U ES ÉTHIQ UES
}

\author{
MARIA GIULIA DONDERO
}

$C_{\text {e }}$

numéro de Protée souhaite proposer une réflexion sur la description - et la descriptibilité - de la subjectivité par le biais d'une étude sur l'éthique. Ce numéro vise plus précisément un double objectif: tout d'abord, rendre compte d'un changement épistémologique à l'intérieur de la discipline sémiotique, de plus en plus intéressée à analyser des pratiques plutôt que des textualités, et, ensuite, décrire comment ce changement épistémologique est fondamentalement lié à une nouvelle approche du sujet humain. Comme le postule la majorité des textes recueillis ici, le sujet ne se caractérise plus seulement comme une position syntaxique à l'intérieur d'une narration clôturée, mais il est analysé en tant que subjectivité pleine en train de se constituer et de s'interroger sur elle-même. Par le biais d'une étude sur l'éthique, qui est le domaine où la subjectivité se manifeste dans toute sa nature problématique, la sémiotique se confronte aux relations entre le sujet conçu en tant que produit discursif et le sujet en tant que personne qui agit dans le monde: elle vise en somme à analyser la dynamique identitaire entre l'expérience in vivo et l'expérience racontée.

Si la théorie greimassienne des années 1990 a témoigné une attention, même timide, au sujet moral (sujet du devoir-être et des valeurs partagées), aucune attention n'a été consacrée au domaine de l'éthique (sujet du pouvoir-être et de la valence «locale» des valeurs). Plus que tout autre, il demande un point de vue différent par rapport à l'approche de l'immanence textuelle et de la théorie narrative classique, parce qu'il s'agit d'un domaine axiologique qui, par définition, laisse ouvert le questionnement sur le sens dans le temps. Ce questionnement sur les fondements du sens doit être saisi tout au long des pratiques «en acte»; pour rendre compte de cette constitution du sens et $d^{\prime}$ une auto-interrogation constante du sujet éthique sur le bien-être de ses actions et décisions, la sémiotique est censée compléter son point de vue analytique avec des approches herméneutique et phénoménologique.

Toutes ces questions décisives sont traitées magistralement dans l'article de Jacques Fontanille qui ouvre le numéro. Ici, I'auteur affirme non seulement que, pour pouvoir parler d'éthique - sans la réduire à l'idéologie -, il faut choisir un autre niveau de pertinence que celui du texte et aborder la question sous l'angle des pratiques, mais aussi qu'il faut dépasser une analyse limitée aux contenus axiologiques pour aborder l'ethos, qui est la forme syntagmatique régulière, reconnaissable et évaluable des pratiques. Fontanille choisit la pensée rhétorique de Perelman pour expliquer comment on peut passer, pour accéder à l'éthique, du paradigme du texte au paradigme de la pratique: les deux schèmes argumentatifs de Perelman que sont la liaison et la dissociation (déliaison, dans les termes de Fontanille) - qui s'appliquent aux relations entre la personne, l'acte 
et le discours -, permettent à l'auteur d'aborder les transformations de l'ethos. L'éthique devient pour Fontanille l'ensemble des opérations portant sur les «liens axiologiques» existant entre ces trois instances (ethos de I'orateur, scénarisation, argument énoncé) auxquelles Fontanille ajoute l'objectif (le résultat) et l'horizon stratégique (la pratique sur laquelle l'acte a des retombées). L'auteur explique que la valeur des pratiques éthiques dépend non pas de l'ethos de l'opérateur, mais de l'ethos de la scène praxique toute entière, c'est-àdire de la totalité des liens constitutifs de la scène à analyser. Fontanille se consacre à l'identification de la nature et des modulations des liens entre ces instances qui se constituent en totalité: la rupture, qui inverse le mode de raisonnement et fait passer de la liaison à la déliaison et réciproquement, d'une part, et le freinage, qui affaiblit la liaison ou la déliaison, d'autre part. À travers ces mouvements, Fontanille décrit la « consistance» de la scène, consistance qui assure la reconnaissance (stabilisation iconique) et le fonctionnement de la scène praxique. $L^{\prime}$ «éthologie» se configure enfin comme l'étude des variations plastiques à l'intérieur de la consistance iconique de cette scène dont les variations et tensions mettent à l'épreuve sa «bonne forme».

Cette réflexion entre en résonance avec les questions présentes dans l'étude de Dondero, qui s'interroge sur la reconnaissance iconique de la pratique éthique et sur les tensions entre fermeture et clôture, entre programmation des actions et impossibilité d'en épuiser le sens. L'article de Dondero thématise en effet la question des forces en jeu à l'intérieur d'une «bonne forme actantielle», notamment à l'intérieur de la scène du pardon. L'auteure part de la problématisation du point de vue requis par la sémiotique pour aborder l'éthique, vu l'impossibilité de la décrire comme une forme dont les bordures des actions sont définies à l'avance comme c'était le cas dans le paradigme du texte. Les pratiques sont en effet des séries de solutions stratégiques en devenir et elles demandent à l'analyste de passer de la pertinence sémiotique de la configuration à la pertinence de l'activité configurante. Comme chez Basso Fossali, on postule ici que la pratique assume toujours le statut d'un scénario décisif pour quelqu'un et que, au cœur de l'éthique des pratiques, il y a la décision, la prise d'initiative d'un sujet.

Ce texte touche différentes questions-clés de la problématique éthique, comme l'autoconscience et l'autoréflexivité du sujet, mais il rentre aussi dans le vif du rapport entre autoréflexion et institution étant donné qu'il s'agit ici de discuter une pratique qui est tout à fait en dehors de toute pratique, le pardon étant un acte isolé, «surabondant» et paradoxal - qui néanmoins se confronte toujours à des questions institutionnelles. En effet, même si le pardon répond d'une logique de l'hors mesure et de l'excès, l'acte de pardonner est toujours soumis au jugement de l'opinion publique et, par conséquent, en négociation et en conflit constants avec la « finitude» de la justice. Sur la relation entre point de vue du sujet et point de vue de l'institution, l'attention de l'étude est donc portée sur le jugement de la doxa; même si l'éthique est un domaine qui se constitue à partir d'une "autorité intérieure», ce n'est pas pour autant qu'elle concerne un discours privé: il faut au contraire une reconnaissance réciproque et publique de la culpabilité, ou du pardon, qui inclue des sujets «tiers» et qui permette d'intégrer dans la mémoire collective les différentes formes de pardon. L'article vise à définir le pardon en le plaçant entre le cadre institutionnel et la recherche, pour tout sujet, du «caractère sensé» de ses propres actions, entre une axiologie conventionnelle et socialisée construite sur les normes morales (procédure et protocole) et la perception locale de la valeur («sagesse en situation » et dramatisation de la décision ad hoc).

L'auteure se consacre enfin à distinguer entre les différentes pratiques de réponses à une offense en décrivant la vengeance, la sanction-réhabilitation, la grâce, I'amnistie et la prescription. Dondero explique comment, à la différence de la prescription et de l'amnistie, le pardon ne peut ni ne doit servir d'instrument 
d'oubli; I'acte du pardon consiste à délier le coupable de son acte, en le considérant comme un «sujet autre que celui qui a commis l'acte».

Cet article non seulement décrit la syntaxe actantielle des gestes du pardon, mais il pointe du doigt le fait que l'efficacité de cet acte dure, parce qu'il exige une bonne tenue des valeurs dans le temps; pour cette raison, le pardon ne peut pas être lu comme en opposition temporelle avec la promesse, ainsi que l'ont fait plusieurs chercheurs: le pardon et la promesse concernent une «conservation dans l'échange» entre passé et futur et le pardon, qui nous engage au futur, se configure lui aussi comme une promesse.

L'article de Bertrand porte sur les rapports problématiques entre sens éthique et émotion et vise notamment la construction d'un ordre syntagmatique de relations entre les deux. Le défi de l'article est en effet de montrer que, à la différence de la plupart des paradigmes philosophiques qui fondent une éthologie où le paramètre émotionnel fait figure d'absent (ou bien se caractérise comme un moment de passivation, comme chez Kant), l'émotion est présente au cœur de l'éthique et précisément à trois moments: à la source de la pensée éthique, dans l'exécution même de l'acte et dans l'aval du discours. Bertrand passe à travers l'explication du processus d'individuation du sujet comme il a été décrit par Simondon pour démontrer que l'émotion assure la relation entre le substrat affectif pré-individuel et l'individuation dans le trans-individuel. À travers la conjonction transductive de l'émotion et de l'action, le sujet simondonien dépasse ses propres limites et se relie respectivement au pré-individuel et au trans-individuel. L'émotion est donc au cœur de l'action et du rapport avec l'autre, comme le montre aussi la réflexion de Bertrand sur I' « éthique sensitive » de Jean-Jacques Rousseau, illustrée dans les Rêveries du promeneur solitaire, qui pose le problème de la responsabilité et du refus de l'imputation de l'acte au nom de l'émotion. Cette éthique sensitive nie l'horizon d'une éthique de l'idéalité (le bien/le mal) en faveur d'une éthique de l'altérité qui reconnaît les axiologies comme fondées sur l'émotion. Plus précisément, la prise en compte de la «Sixième Promenade» de Rousseau vise à démontrer que la perspective égocentrée de l'émotion fonde un sens éthique: I'émotion se montre coextensive à l'action ellemême, lui conférant sens et valeur parce qu'elle instaure les conditions d'une co-responsabilité avec l'autre. La relation entre émotion et relation avec l'autre dans l'action est approfondie lors de la description de la passerelle entre éthique et morale, entre le téléologique, fondé sur la réversibilité émotionnelle avec autrui, et le déontologique, fondé sur l'obligation et l'inauthenticité du sentir. La pratique éthique se caractérise en effet comme la fusion du sujet dans son acte: la composante émotionnelle, responsable du lien transductif qui s'établit entre toutes les instances de la scène, assure l'unité du sujet. Dans la conduite morale, au contraire, l'inhérence du sujet à son acte peut devenir répétition et enfin protocole, et c'est là que le sujet se sépare en une pluralité $d^{\prime}$ instances et se disjoint de lui-même. Bertrand montre de manière très fine le changement de statut de l'émotion du sujet, lorsque celle-ci disparaît et ne laisse la place qu'à l'exécution des normes et à une individualité déviée.

Basso Fossali, tout comme Bertrand, traite la question de l'émotion éthique à partir d'une réflexion sur I'auto-destination et l'auto-réflexion. En effet, comme le constate l'auteur, même si l'éthique est ancrée dans une observation de deuxième ordre, la réflexion éthique retrouve une sémantisation sans monitorage d'ellemême dans les émotions. L'article de Basso Fossali reprend aussi la question épistémologique d'une sémiotique des pratiques qui, trouvant dans l'éthique son axe focal, démontre son irréductibilité à la textualité. Le sens éthique, en effet, ne peut pas se réduire à une configuration textuelle donnée, étant donné que le domaine de 
I'éthique concerne une pratique inépuisable de reconfigurations narratives à gérer dans le temps, qui vise à combler l'hétérogénéité de l'expérience. L'éthique est donc définie comme une constante expérimentation identitaire et comme la négociation de l'agir sensé qui dépasse les garanties de sens offertes par les domaines sociaux. L'auteur montre que l'éthique, étant subversive par rapport aux rôles identitaires déjà établis, garde une paradoxale affinité avec la créativité, créativité qui permet au sujet de chercher une solution « toujours ad hoc » "pour reconstruire une commensurabilité entre scénarios d'ancrage de valeurs totalement irréductibles et inconséquentes». Ce travail de Basso Fossali met au centre de la question éthique la personnalité des acteurs sociaux, qui est la capacité à gérer des rôles identitaires divers face à soi-même et face à autrui. Cette personnalité se caractérise non pas à travers la «liberté», qui répondrait déjà d'une idéologie, et donc d'une morale, mais à travers une ouverture enracinée dans l'expérimentation du sens «ultérieurement possible». La question de la personnalité est donc au cœur de la distinction entre morale et éthique: I'éthique émerge lorsque la suture narrative de la personnalité ne peut pas compter sur des rôles moralisés déjà socialisés; en effet, la personnalité «ouverte» est toujours mise à l'épreuve (créativité) par les significations expérientielles les plus différentes - qu'elle est censée totaliser. Sur le plan social, l'éthique se dessine pour Basso Fossali à la fois comme une demande constante de traduction entre valeurs et destins différents et comme la constatation du caractère intraduisible des personnalités: la commensurabilité souhaitée entre destins est contrebalancée par une expansion/densification des scénarios identitaires et moraux concurrentiels. Basso Fossali propose une possible solution à cette impasse du sujet éthique: à l'inachèvement personnel, on peut donner comme réponse le croisement des destins, bien au-delà de la descendance, de l'héritage familial et national: «le sentiment d'incomplétude peut être comblé à travers la clôture asymptotique de l'asymétrisation individualisante, à travers la vocation de mettre son propre héritage à la recherche d'une "famille" ».

L'article de Panier vise lui aussi à distinguer entre morale et éthique et entre deux modèles de sujet et d'investissement des valeurs. Dans le modèle de la sémiotique classique, axiologie et idéologie déterminent le champ des valeurs où un sujet trouve à se définir; en revanche, le modèle «éthique » devrait concerner plutôt le sens de l'action et sa fonction dans la constitution d'un sujet: la question éthique concerne en effet le cours d'action par lequel un sujet peut se définir, et pas seulement la jonction d'un sujet à un objet de valeur. La problématisation de l'éthique se joue donc entre les valeurs d'objet et les valeurs d'action, valeurs qui se manifestent comme concurrentielles dans un récit-parabole, extrait de l'Évangile de Luc, que Panier choisit pour répondre à la question centrale de sa réflexion: "y a-t-il, en deçà (ou au-delà) de la position des valeurs, une place pour la convocation et l'instauration d'un sujet?». Ce récit-parabole manifeste ce qu'il en est du statut des sujets en fonction des rapports aux valeurs investies dans les objets (leur pouvoir-faire ou savoirfaire), d'une part, et au parcours d'action (l'instauration des sujets et les conditions de leur engagement dans un parcours), d'autre part. Dans la parabole, l'objet engage le sujet impliqué non pas pour sa valeur matérielle, mais pour le statut fiduciaire décisif que, par son biais, le sujet engendre avec autrui. Le récit choisi par Panier montre, comme le font les articles de Basso Fossali et Dondero, que la pratique éthique est paradoxale: ici, le récit-parabole nous conduit vers un dispositif caractérisé par la non-valeur et le non-signe de l'objet (l'objet n'est pas convertible en valeur matérielle). Ce dispositif marque l'importance de la singularité d'un sujet dans l'acte énonciatif qui l'instaure et d'un système intersubjectif et énonciatif caractérisé par une rationalité figurale qui fait écart à la rationalité pratique. Les questions éthiques se caractérisent en effet comme celles qui concernent le point d'émergence des valeurs pour un sujet et la valeur des valeurs. La question éthique consiste enfin dans 
l'écart entre le statut du sujet par rapport à l'univers de valeurs (idéologique) et le statut du sujet qui se dessine par rapport au parcours en quoi se déploie une pratique, un point d'écart qui dissocie la structure idéologique du signe (où l'objet serait le signifiant de la valeur) des expériences intersubjectives et énonciatives du sujet. Cette tension a également été explorée au sujet de la pratique du sémioticien engagé dans la construction d'une cohérence de discours dont il fait l'expérience. La posture de l'interprète peut être investie d'une compétence encyclopédique, qui déploie la signification des objets dans la reconnaissance de réseaux conformes à la sémiotique du monde naturel et qui le fait rester au seuil du parcours de l'interprétation; la posture de l'interprète peut également se configurer comme idéologique, apte à assumer les univers de valeurs à l'œuvre dans le discours qui manifeste la cohérence d'un système de valeurs investies dans des dispositifs narratifs; mais la posture la plus profonde est celle que Panier définit comme langagière (ou littérale), selon la définition de Lacan. Paradoxalement, cette posture littérale s'atteste et se joue dans la figure (et en figures), dans un supplément de sens typiquement rhétorique qui dessine un excès et un écart par rapport aux valeurs et aux formes des systèmes axiologiques.

L'article de Kremer-Marietti porte sur les fondements éthiques de la constitution du sujet, sujet qui se caractérise en tant que moi à travers la rencontre du monde que l'autre lui offre. Le rapport à autrui se configure comme un rapport externe d'un moi à l'autre qui se transforme en un rapport interne au moi réel - qui lui permet en même temps d'accéder à une connaissance de l'autre et de sa perspective propre. Dans le monde réel de la société, le socius (l'autre en tant qu'ami) joue un rôle indispensable dans l'action morale parce qu'il oblige le moi à essayer de distinguer la connaissance de soi proprement dite et l'obligation morale de se connaître afin de s'orienter dans l'action à partir de la connaissance que l'autre a de lui-même. L'éthique se constitue par ce jeu de miroirs qui met en partage différentes "connaissances de soi», en intensifiant les éléments d'échange entre les différentes personnalités ainsi confrontées. C'est pour cela que le rapport à l'autre apparaît non plus comme un rapport externe d'un moi à l'autre, mais comme un rapport interne au moi réel. La perspective éthique est donc au cœur de la naissance du sujet, qui est toujours suscité par autrui: le face à face qui caractérise la formation d'un sujet est déjà profondément éthique. À ce propos, l'auteure décrit la statique de la personnalité comme reposant sur l'instance de la réceptivité, de la sensibilité, de l'émotivité, qui se dessine à travers une ouverture sensible constante sur le monde et sur autrui. Une dynamique de cette personnalité est constituée par des pivots d'ordre éthique qui caractérisent la rencontre du sujet avec le monde, telles la responsabilité et la liberté, et qui nous confrontent depuis le début de notre existence à une culpabilité fondatrice. Le sens éthique se caractérise encore une fois comme vécu intérieurement, bien au-delà de ce qui peut être objectivement et socialement constaté. À ce sujet, l'auteure définit le droit comme témoin de la perte de l'innocence et la culpabilité comme fondement du pouvoir politique. En suivant les enseignements platoniciens, l'auteure distingue entre aidos - qui se caractérise comme l'honneur, la pudeur ou même la honte, et qui constitue le premier principe apte à nous rendre réflexifs quant à notre mérite - et dikè, la justice. Les deux notions sont grandement liées étant donné que la dignité du sujet (autonomie créatrice) dépend aussi des obligations décrétées par la justice. Au cœur de l'éthique demeure en somme une confrontation avec la société et une prise de conscience constante de soi-même.

Enfin, l'article de Laugier propose une profonde réflexion en trois temps sur l'éthique en partant de la seconde philosophie de Wittgenstein. Tout d'abord, il s'agit pour l'auteure de dessiner le projet d'une éthique 
"sans ontologie», qui s'occupe d'explorer les pratiques et l'immanence de notre vie ordinaire; ensuite, il s'agit de caractériser cette exploration à travers la capacité, que le sujet doit acquérir, de saisir ce qui est important et ce qui compte pour lui (matter); et enfin il s'agit de comprendre la perception morale comme une aventure identitaire.

En partant de l'approche wittgensteinienne qui se fonde sur la constatation qu'il n'y a pas d'objet ou de sujet spécifique de la morale, cette éthique «sans sujet particulier» se caractérise comme une éthique de la perception, qui dépend de notre capacité à voir, à être attentifs et attentionnés envers nous-mêmes et notre expérience: "La capacité à percevoir le détail de la vie ordinaire - saisir "ce qui compte" sur l'arrière-plan de la forme de vie - est un élément central de la compétence morale». Cette manière d'entendre l'éthique est bien éloignée du champ de la logique et de l'argumentation; elle se fonde plutôt sur une éducation du sensible. Pour l'auteure, l'éthique se caractérise comme la capacité à une exploration perceptive, sensible et conceptuelle, aux détails et expressions morales qui sont analysables à travers nos pratiques de langage quotidien. Cette étude montre de manière magistrale que la littérature, plus que la philosophie, est le champ d'exploration privilégié de cette éthique parce qu'elle nous permet de nous apercevoir du détachement du détail et du geste sur l'arrière-plan d'une forme de vie. Cet arrière-plan nous permet de voir clairement nos actions et les actions $d^{\prime}$ 'autrui et d'avoir une perception de ce qui est signifiant pour nous. Cette approche gestaltiste en morale fait ressortir une texture d'être des sujets qui a à voir non pas avec leurs choix moraux, mais avec «ce qui importe» et qui exprime les différences entre individus. La notion de «texture perceptive de la vie», encore plus précisément que la notion de figure/fond, désigne une réalité instable qui, loin d'être fixée par des concepts, peut être saisie à travers la reconnaissance de l'émergence de gestes, de manières, de styles. L'attention pour les styles de chacun caractérise cette éthique comme une éthique du particulier, centrée sur les visions particulières et les «configurations» de pensée des individus.

Cette dimension de la morale se dessine enfin comme aventure de la personnalité à la fois conceptuelle (c'est une manière d'étendre ses concepts) et sensible (on expose toute notre vulnérabilité). Une telle aventure requiert des qualités spécifiques comme celle de l'improvisation morale. La littérature, en ce sens, pousse à la limite nos capacités de compréhension des actions et paroles d'autrui perçues comme incompréhensibles. On retrouve ici le caractère intraduisible entre personnalités et destins, sur lesquels l'article de Basso Fossali s'est arrêté longuement. C'est, en effet, dans ce que Laugier appelle «improvisation morale » et capacité d'arriver à comprendre l'abandon de la part de l'autre du sens commun (que nous percevons comme non-sens de l'autre) qui devrait nous permettre d'être prêts à « perdre nos concepts» et nos pratiques pour les projeter ailleurs, dans «des contextes nouveaux» qui pourraient nous faire poursuivre l'aventure. 